\title{
NONLINEAR ANALYSIS OF TORSIONALLY LOADED PILE GROUPS
}

\author{
L. G. KONG ${ }^{\text {i) }}$ and L. M. ZHANG ${ }^{\text {ii) }}$
}

\begin{abstract}
An empirical approach is developed to analyze the nonlinear torsional behavior of free-standing pile groups with rigid pile caps. In this approach, the lateral and torsional responses of individual piles in a pile group are modeled by $p-y$ and $\tau-\theta$ curves; the interaction among lateral resistances of the individual piles is predicted through Mindlin's elastic solutions; the interactions between the torsional and lateral resistances of the individual piles are described through Randolph's solution; and the coupling effect of lateral resistance on torsional resistance of the individual piles is quantified using an empirical factor " $\beta$ ". The proposed approach is capable of capturing the most significant aspects of pile-soil-pile interactions and coupling effect in pile groups subjected to torsion. The proposed approach is verified using results of centrifuge model tests. In general, the applied torque-twist angle response and the transfer of applied torque in pile groups can be reasonably well predicted and are sensitive to the pile group configuration.
\end{abstract}

Key words: centrifuge tests, nonlinear analysis, pile foundations, pile group, pile-soil-pile interaction, torsional response (IGC: E12)

\section{INTRODUCTION}

Foundations for offshore platforms, bridge bents, and tall buildings are subjected to significant torsional loads by virtue of eccentric lateral loading from wind and wave action, ship impacts or high-speed vehicles. In the past, researchers have developed numerical solutions for single piles subjected to torsion (e.g., Poulos, 1975; Randolph, 1981; Chow, 1985; Georgiadis, 1987; Guo and Randolph, 1996). Zhang and Tsang (2005) studied the behavior of a torsionally loaded $2 \times 2$ bored pile group using a threedimensional finite difference method. Recently, Kong (2006) and Kong and Zhang (2007b) reported a series of centrifuge model tests to investigate load sharing mechanisms and pile-soil-pile interactions in threediameter spaced $1 \times 2,2 \times 2$ and $3 \times 3$ fixed-head pile groups subjected to torsion. From these studies, the following attributes of torsionally loaded pile groups were observed:

1. A pile group subjected to torsion simultaneously mobilizes lateral and torsional resistances of individual piles, as shown in Fig. 1. The torsional resistances resist $20 \sim 50 \%$ of the applied torque; the mobilization of lateral resistance is closely related to pile locations within the group and pile-soil-pile interactions.

2. The effect of horizontal movement of a pile on the lateral behavior of its adjacent piles or "shadowing" effect is also present in the pile groups subjected to torsion. However, the interaction effects in pile groups subjected to torsion and lateral loading are significantly different, because the directions and magnitudes of horizontal movements of individual piles in pile groups subjected to torsion are different (see Fig. 1). In addition, the horizontal movement of a pile affects the torsional behavior of its adjacent piles.

3. The lateral soil reaction on a pile in a pile group tends to increase the torsional resistance of the pile, which is referred to as deflection-torsion coupling effect.

To date, few computer programs for routine pile design simulate the behavior of pile groups subjected to torsion considering the pile-soil-pile interactions (also known as "PSPI") and the deflection-torsion coupling effect. MPILE, a program originally developed by Randolph (1980) under the name of PIGLET, uses approximate analytical solutions for the lateral and torsional stiffnesses of individual piles in a group, and takes into account the effect of lateral resistances of the individual piles on the torsional stiffness of the pile group using interaction factors. The program is considered applicable for the analysis of pile groups under small deformations. GROUP (Reese et al., 2000) and FB-Pier (Hoit et al., 2001), based on Winkler's spring idealization of soil, employ load-transfer functions to represent the relationship between a load at any point along a pile and the associated soil deformation at that point. The load-transfer approach has been widely adopted for routine design, especially where nonlinear soil behavior has to be considered and/or soil stratification is complex. In the load-transfer

i) Associate Professor, MOE Key Laboratory of Soft Soils and Geoenvironmental Engineering, Zhejiang University, China (klg@zju.edu.cn) (formerly Postdoctoral Research Associate, Hong Kong University of Science and Technology).

ii) Associate Professor, Hong Kong University of Science and Technology, Hong Kong (cezhangl@ust.hk). The manuscript for this paper was received for review on April 3, 2008; approved on January 29, 2009.

Written discussions on this paper should be submitted before November 1, 2009 to the Japanese Geotechnical Society, 4-38-2, Sengoku, Bunkyo-ku, Tokyo 112-0011, Japan. Upon request the closing date may be extended one month. 

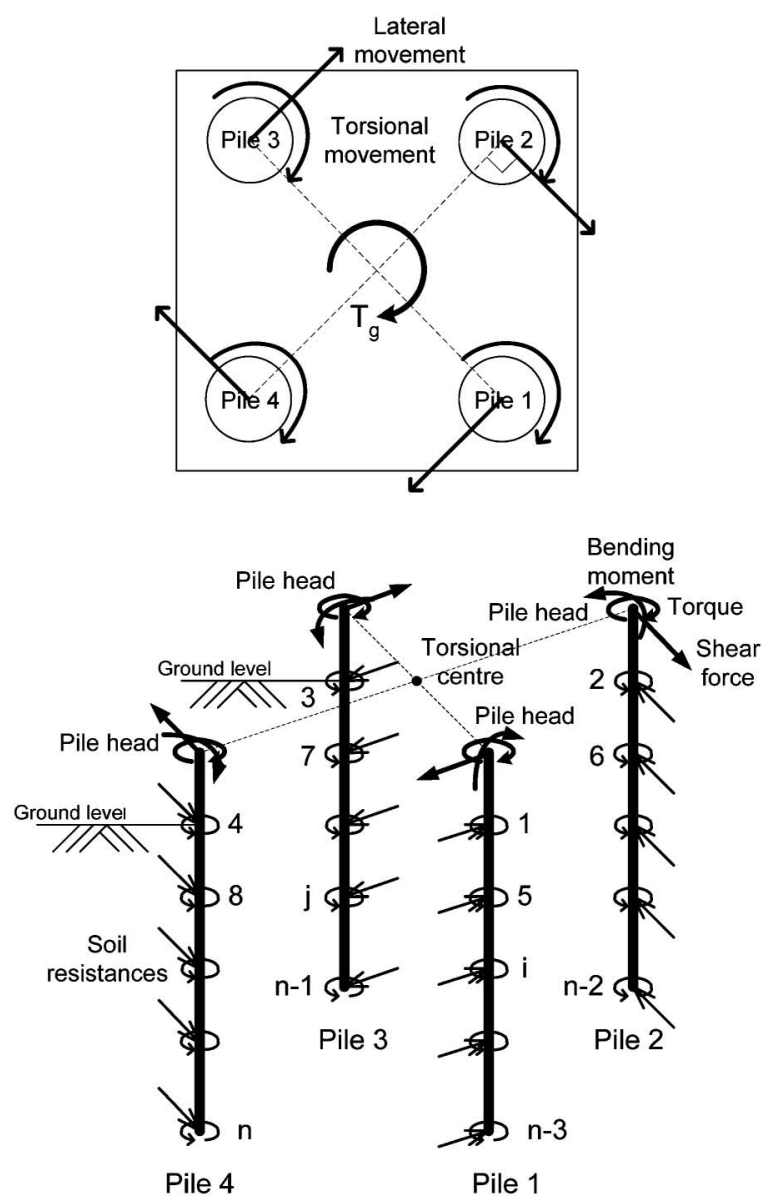

Fig. 1. Load distribution in a pile group subjected to torsion and discretization of piles

approach, pile-soil-pile interactions are taken into account using empirical factors, which are usually obtained by back-calculating pile load test results. For example, to analyze laterally loaded pile groups, a $p$-multiplier factor, first suggested by Brown et al. (1988), is employed in GROUP and FB-Pier to quantify the loss of soil resistance due to "shadowing" effects (i.e., the geometric nonlinearity that influences the lateral response of a pile as lateral support is withdrawn from the soil in front of that pile as the pile positioned forward of it moves in the same direction as the piles in the group). As reported by Kong (2006), "shadowing" effects in pile groups subjected to torsion and lateral loading are significantly different, so empirical factors back-calculated from tests on laterally loaded pile groups may not be applicable to torsionally loaded pile groups.

This paper describes a rational numerical approach to take into account several attributes of torsionally loaded pile groups; namely, (1) nonlinear behavior of soil adjacent to piles in pile groups subjected to torsion, (2) pilesoil-pile interactions, and (3) the deflection-torsion coupling effect. Formulations for the approach will be presented first. Next, the experimental results reported by Kong (2006) and Kong and Zhang (2007b) are used to verify the accuracy of the proposed approach. Finally, influences of the pile-soil-pile interactions and the deflec-

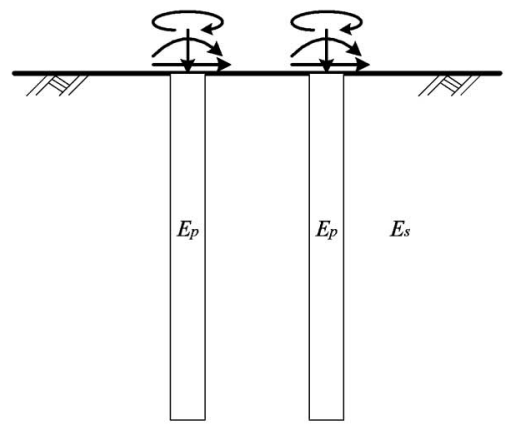

(a) Problem

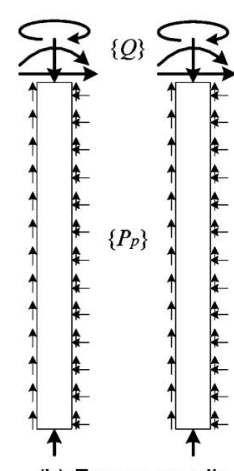

(b) Forces on pile

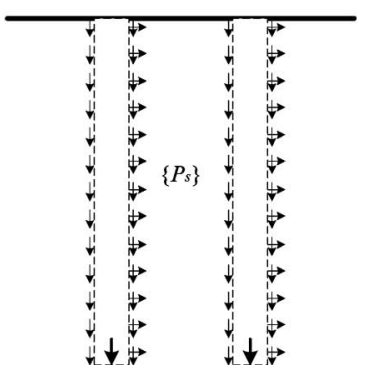

(c) Forces on soil
Fig. 2. Pile-soil-pile interactions

tion-torsion coupling effect on response of pile groups are studied.

\section{PILE-SOIL SYSTEM}

A pile-soil-pile interaction problem, as represented in Fig. 2, can be decomposed into two domains; namely (1) the pile domain, i.e., the group piles subjected to external loads $\{Q\}$ and pile-soil interaction forces acting on the piles, $\left\{P_{\mathrm{p}}\right\}$;

(2) the soil domain, i.e., the soil mass acted on by a system of pile-soil interaction forces $\left\{\boldsymbol{P}_{\mathrm{s}}\right\}$, at the boundary of the pile-soil interface.

The pile domain and the soil domain interact with each other through the pile-soil interaction forces, as shown in Fig. 2, and the compatibility of the deformations of the soil and pile domains.

\section{Pile Domain}

The pile shafts are assumed to be linear elastic and obey the small deformation assumption; the piles are vertical and fixed to the pile cap; the pile toes are subjected to torsional and vertical restraints; and the pile cap is rigid and not in contact with the ground. Thus, an individual pile in a pile group under torsional loading is subjected to a lateral load, a bending moment, and a torsional load at the pile head (see Fig. 1). Assuming each pile works as a simple beam, the governing fourth and second order differential equations for lateral deflection $y$ and twist angle $\theta$ of the pile can be given by, 


$$
\begin{aligned}
& E_{\mathrm{p}} I_{\mathrm{p}} \frac{\partial^{4} y}{\partial z^{4}}+k_{\mathrm{h}} y=0 \\
& G_{\mathrm{p}} J_{\mathrm{p}} \frac{\partial^{2} \theta}{\partial z^{2}}-k_{\theta} \theta=0
\end{aligned}
$$

where $E_{\mathrm{p}}$ and $G_{\mathrm{p}}$ are the elastic modulus and shear modulus of pile shaft, respectively; $I_{\mathrm{p}}$ and $J_{\mathrm{p}}$ are the second moment and polar second moment of area of pile section, respectively; $z$ is depth; and $k_{\mathrm{h}}$ and $k_{\theta}$ are the moduli of subgrade reaction for lateral loading and torsional loading, respectively. Each pile is modeled by a number of discrete beam elements. Figure 1 shows the node numbering method employed in the proposed approach. Based on the finite element method (e.g., Smith, 1982), the loaddeformation relationship is written as

$$
\left[K_{\mathrm{p}}\right]\left\{W_{\mathrm{p}}\right\}=\{Q\}+\left\{P_{\mathrm{p}}\right\}
$$

where $\left[K_{\mathrm{p}}\right]$ is the global stiffness matrix of all elements of the group piles; $\left\{W_{\mathrm{p}}\right\}$ is the vector of deformations at the pile nodes.

\section{Soil Domain}

It is assumed that nonlinear behavior of a pile group is due to the nonlinear soil response in the near field (i.e., at individual piles in the group, represented by $p-y$ and $\tau-\theta$ curves, where $p$ is the lateral soil reaction; $y$ is the lateral pile deflection; $\tau$ is the torsional shear stress; and $\theta$ is the local twist angle of pile shaft) but the far-field interactions (i.e., pile-soil-pile interactions) are linear elastic. In the present approach, a "lumped" formulation in which soil stiffness is lumped at the pile nodes is adopted, which is adequate in most practical problems. Thus, the soil deformation at node $i$ due to its own loading as well as loadings at other nodes, $\boldsymbol{\varpi}_{\mathrm{si}}$, can be obtained by superposition:

$$
\boldsymbol{\varpi}_{\mathrm{si}}=\sum_{\mathrm{j}=1}^{\mathrm{n}} f_{\mathrm{ij}} P_{\mathrm{sj}}
$$

where $f_{\mathrm{ij}}$ is the flexibility coefficient denoting the deformation at node $i$ due to a unit load at node $j ; P_{\mathrm{sj}}$ is the pilesoil interaction force acting on the soil at node $j$; and $n$ is the total number of nodes. Equation (4) can be written for each node, leading to the following flexibility relationship for the soil

$$
\left\{W_{\mathrm{s}}\right\}=\left[F_{\mathrm{s}}\right]\left\{P_{\mathrm{s}}\right\}
$$

where $\left\{W_{\mathrm{s}}\right\}$ is the vector of soil deformation; $\left[F_{\mathrm{s}}\right]$ is the soil flexibility matrix; and $\left\{P_{\mathrm{s}}\right\}$ is the vector of pile-soil interaction forces acting on the soil.

In torsionally loaded pile groups, the pile-soil interaction forces acting on the soil at node $j, P_{\mathrm{sj}}$, include a lateral force and a torsional force, denoted as $H_{\mathrm{sj}}$ and $T_{\mathrm{sj}}$, respectively. The corresponding flexibility coefficients are also divided into two parts, denoted as $f_{\mathrm{ij}}^{\mathrm{H}}$ and $f_{\mathrm{ij}}^{\mathrm{T}}$. Thus, Eq. (4) can be decomposed as,

$$
\boldsymbol{\varpi}_{\mathrm{si}}=\sum_{\mathrm{j}=1}^{\mathrm{j}=\mathrm{n}}\left(f_{\mathrm{ij}}^{\mathrm{H}} H_{\mathrm{sj}}+f_{\mathrm{ij}}^{\mathrm{T}} T_{\mathrm{sj}}\right)=\sum_{\mathrm{j}=1}^{\mathrm{j}=\mathrm{n}}\left[f_{\mathrm{ij}}^{\mathrm{H}} f_{\mathrm{ij}}^{\mathrm{T}}\right]\left\{\begin{array}{c}
H_{\mathrm{sj}} \\
T_{\mathrm{sj}}
\end{array}\right\} .
$$

Since the soil deformation at node $i, \boldsymbol{\varpi}_{\mathrm{si}}$ in this case, includes a lateral component, $\boldsymbol{\varpi}_{\text {si }}^{\mathrm{H}}$, and a torsional component, $\boldsymbol{\varpi}_{\mathrm{si}}^{\mathrm{T}}$, Eq. (6) is further decomposed as,

$$
\left\{\begin{array}{c}
\boldsymbol{\varpi}_{\mathrm{si}}^{\mathrm{H}} \\
\boldsymbol{\varpi}_{\mathrm{si}}^{\mathrm{T}}
\end{array}\right\}=\sum_{\mathrm{j}=1}^{\mathrm{j}=\mathrm{n}}\left[\begin{array}{ll}
f_{\mathrm{ij}}^{\mathrm{HH}} & f_{\mathrm{ij}}^{\mathrm{HT}} \\
f_{\mathrm{ij}}^{\mathrm{TH}} & f_{\mathrm{ij}}^{\mathrm{TT}}
\end{array}\right]\left\{\begin{array}{c}
H_{\mathrm{sj}} \\
T_{\mathrm{sj}}
\end{array}\right\}
$$

where $f_{\mathrm{ij}}^{\mathrm{HH}}$ and $f_{\mathrm{ij}}^{\mathrm{TH}}$ are the two components of $f_{\mathrm{ij}}^{\mathrm{H}} ; f_{\mathrm{ij}}^{\mathrm{HT}}$ and $f_{\mathrm{ij}}^{\mathrm{TT}}$ are the two components of $f_{\mathrm{ij}}^{\mathrm{T}}$. The physical meaning of each of these flexibility coefficients is:

$f_{\mathrm{ij}}^{\mathrm{HH}}$ : the lateral component of the soil deformation at node $i$ due to a unit lateral force at node $j$;

$f_{\mathrm{ij}}^{\mathrm{TH}}$ : the torsional component of the soil deformation at node $i$ due to a unit lateral force at node $j$;

$f_{\mathrm{ij}}^{\mathrm{HT}}$ : the lateral component of the soil deformation at node $i$ due to a unit torsional force at node $j$;

$f_{\mathrm{ij}}^{\mathrm{TT}}$ : the torsional component of the soil deformation at node $i$ due to a unit torsional force at node $j$.

In terms of sources of loading, the soil deformation at a node consists of the one due to the forces at the node and the one due to the forces at other nodes. So $\boldsymbol{\varpi}_{\mathrm{si}}^{\mathrm{H}}$ and $\boldsymbol{\varpi}_{\text {si }}^{\mathrm{T}}$ are each further divided into two components:

$$
\begin{aligned}
& \boldsymbol{\varpi}_{\mathrm{si}}^{\mathrm{H}}=\boldsymbol{\varpi}_{\mathrm{sii}}^{\mathrm{H}}+\boldsymbol{\varpi}_{\mathrm{ai}}^{\mathrm{H}} \\
& \boldsymbol{\varpi}_{\mathrm{si}}^{\mathrm{T}}=\boldsymbol{\varpi}_{\mathrm{sii}}^{\mathrm{T}}+\boldsymbol{\varpi}_{\mathrm{ai}}^{\mathrm{T}}
\end{aligned}
$$

where $\boldsymbol{\varpi}_{\text {sii }}$ represents the soil deformation at node $i$ as a result of forces at the same node; $\boldsymbol{\varpi}_{\text {ai }}$ represents the added soil deformation at node $i$ as a result of forces at nodes other than $i$. Referring to Eq. (7), Eqs. (8) and (9) can be rewritten as

$$
\begin{aligned}
\boldsymbol{\varpi}_{\mathrm{si}}^{\mathrm{H}} & =\boldsymbol{\varpi}_{\mathrm{sii}}^{\mathrm{H}}+\boldsymbol{\varpi}_{\mathrm{ai}}^{\mathrm{H}} \\
& =\left(f_{\mathrm{ii}}^{\mathrm{HH}} H_{\mathrm{si}}+f_{\mathrm{ii}}^{\mathrm{HT}} T_{\mathrm{si}}\right)+\sum_{\substack{\mathrm{j}=1 \\
\mathrm{j} \neq \mathrm{i}}}^{\mathrm{j}=\mathrm{n}}\left(f_{\mathrm{ii}}^{\mathrm{HH}} H_{\mathrm{sj}}+f_{\mathrm{ii}}^{\mathrm{HT}} T_{\mathrm{sj}}\right) \\
\boldsymbol{\varpi}_{\mathrm{si}}^{\mathrm{T}} & =\boldsymbol{\varpi}_{\mathrm{sii}}^{\mathrm{T}}+\varpi_{\mathrm{ai}}^{\mathrm{T}} \\
& =\left(f_{\mathrm{ii}}^{\mathrm{TH}} H_{\mathrm{si}}+f_{\mathrm{ii}}^{\mathrm{TT}} T_{\mathrm{si}}\right)+\sum_{\substack{\mathrm{j}=1 \\
\mathrm{j} \neq \mathrm{i}}}^{\mathrm{j}=\mathrm{n}}\left(f_{\mathrm{ii}}^{\mathrm{TH}} H_{\mathrm{sj}}+f_{\mathrm{ii}}^{\mathrm{TT}} T_{\mathrm{sj}}\right)
\end{aligned}
$$

The matrix form of Eqs. (10) and (11) is in essence the same as Eq. (5). Inherent in the load-transfer approach for modeling soil behavior in a single pile is the assumption that the soil reactions are uncoupled; that is, the displacement at a particular node will only affect the soil reaction at that node. Thus, for loadings at node $j$ which is associated with the same pile as node $i$, and for $j \neq i$, the values of the flexibility coefficients, $f_{\mathrm{ij}}^{\mathrm{HH}}, f_{\mathrm{ij}}^{\mathrm{HT}}, f_{\mathrm{ij}}^{\mathrm{TH}}$ and $f_{\mathrm{ij}}^{\mathrm{TT}}$ are zero. The calculation of the non-zero flexibility coefficients in Eqs. (10) and (11) is presented in the next section.

\section{Flexibility Coefficients of Soil}

$f_{\mathrm{ii}}^{\mathrm{HH}}$ and $f_{\mathrm{ii}}^{\mathrm{TT}}$

$f_{\mathrm{ii}}^{\mathrm{HH}}$ and $f_{\mathrm{ii}}^{\mathrm{TT}}$ are used to model soil response in the near field. $f_{\mathrm{ii}}^{\mathrm{HH}}$ denotes the lateral component of soil deformation at node $i$ due to a unit lateral force at the same node; $f_{\mathrm{ii}}^{\mathrm{TT}}$ is the torsional component of soil deformation at node $i$ due to a unit torsional force at the same node. In this paper, nonlinear $p-y$ and $\tau-\theta$ curves are used to calculate the two flexibility coefficients: 


$$
\begin{aligned}
& f_{\mathrm{ii}}^{\mathrm{HH}}=\frac{1}{k_{\mathrm{h}} \delta} \\
& f_{\mathrm{ii}}^{\mathrm{TT}}=\frac{2}{\pi} \frac{1}{k_{\theta} D^{2} \delta}
\end{aligned}
$$

where $D$ is the pile diameter and $\delta$ is the pile segment length. The soil reaction moduli $k_{\mathrm{h}}$ and $k_{\theta}$, which vary with load, are obtained using $p-y$ and $\tau-\theta$ curves. In the literature, many $p-y$ curves were recommended for different soils (e.g., Reese et al., 1974; Yan and Byrne, 1992; Kong, 2006); several $\tau-\theta$ curves for sand were also proposed (Dutt and O'Neill, 1983; Hoit et al., 2001; Kong, 2006).

\section{$f_{\mathrm{ii}}^{\mathrm{TH}}$ and $f_{\mathrm{ii}}^{\mathrm{HT}}$}

$f_{\mathrm{ii}}^{\mathrm{TH}}$ and $f_{\mathrm{ii}}^{\mathrm{HT}}$ reflect the effect of the lateral force/torsional force on the torsional force/lateral force in an individual pile, which are named 'coupling effect' by Kong (2006). $f_{\mathrm{ii}}^{\mathrm{TH}}$ represents the torsional component of soil deformation at node $i$ due to a unit lateral force at the same node. $f_{\mathrm{ii}}^{\mathrm{HT}}$ represents the lateral component of soil deformation at node $i$ due to a unit torsional force at the same node. Kong (2006) found from centrifuge model tests that the latter effect is minor, so $f_{\mathrm{ii}}^{\mathrm{HT}}$ is ignored in the present approach.

To further clarify the physical interactions behind $f_{\mathrm{ii}}^{\mathrm{TH}}$, $\boldsymbol{\varpi}_{\text {sii }}^{\mathrm{T}}$ in Eq. (11) is rewritten as

$\boldsymbol{\varpi}_{\mathrm{sii}}^{\mathrm{T}}=f_{\mathrm{ii}}^{\mathrm{TH}} H_{\mathrm{si}}+f_{\mathrm{ii}}^{\mathrm{TT}} T_{\mathrm{si}}=\left(1+\frac{f_{\mathrm{ii}}^{\mathrm{TH}} H_{\mathrm{si}}}{f_{\mathrm{ii}}^{\mathrm{TT}} T_{\mathrm{si}}}\right) f_{\mathrm{ii}}^{\mathrm{TT}} T_{\mathrm{si}}=\frac{f_{\mathrm{ii}}^{\mathrm{TT}}}{\alpha_{\mathrm{TH}(\mathrm{i})}} T_{\mathrm{si}}$

where $\alpha_{\mathrm{TH}(\mathrm{i})}$ is a modification factor (or interaction factor) at node $i$ and expressed as

$$
\alpha_{\mathrm{TH}(\mathrm{i})}=\frac{f_{\mathrm{ii}}^{\mathrm{TT}} T_{\mathrm{si}}}{f_{\mathrm{ii}}^{\mathrm{TH}} H_{\mathrm{si}}+f_{\mathrm{ii}}^{\mathrm{TT}} T_{\mathrm{si}}}=1-\frac{f_{\mathrm{ii}}^{\mathrm{TH}}}{\boldsymbol{\varpi}_{\mathrm{sii}}^{\mathrm{T}}} H_{\mathrm{si}} .
$$

If one employs the same numerical technique to analyze the response of a torsionally loaded single pile, the torsional soil deformation at node $i, \boldsymbol{\varpi}_{\mathrm{si}}$, is then

$$
\varpi_{\mathrm{si}}=f_{\mathrm{i}} T_{\mathrm{si}}^{\prime}
$$

where $f_{\mathrm{i}}$ and $T_{\mathrm{si}}^{\prime}$ are the soil flexibility coefficient and the torsional force at node $i$ in the single pile. Assuming $\boldsymbol{\varpi}_{\text {sii }}^{\mathrm{T}}$ $=\boldsymbol{\varpi}_{\mathrm{si}}$ and $f_{\mathrm{ii}}^{\mathrm{TT}}=f_{\mathrm{i}}$, one obtains

$$
T_{\mathrm{si}}=\alpha_{\mathrm{TH}(\mathrm{i})} T_{\mathrm{si}}^{\prime}
$$

Equation (17) reveals that $\alpha_{\mathrm{TH}(\mathrm{i})}$ is the ratio of the torsional force at node $i$ of a group pile to that at the corresponding node on a single pile at the same twist angle. $\alpha_{\mathrm{TH}(\mathrm{i})}$ is a function of $H_{\mathrm{si}}$ (see Eq. (15)). Therefore, $f_{\mathrm{ii}}^{\mathrm{TH}}$ reflects the effect of the lateral force $H_{\mathrm{si}}$ on the torsional force $T_{\mathrm{si}}$ at the same node.

Defining a new coupling coefficient, $\beta$,

$$
\beta=-\frac{f_{\mathrm{ii}}^{\mathrm{TH}}}{\boldsymbol{\varpi}_{\text {sii }}^{\mathrm{T}}} p_{\mathrm{a}} D \delta
$$

where $p_{\mathrm{a}}$ is the atmospheric pressure, Eq. (15) becomes

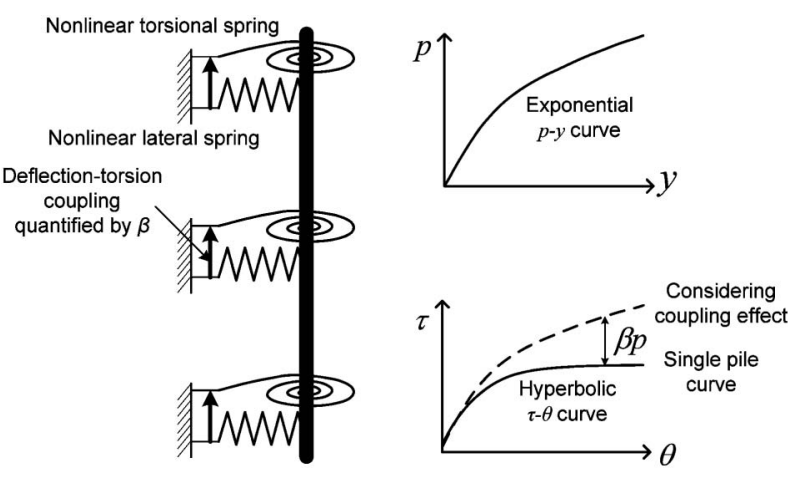

Fig. 3. Modeling of soil responses and deflection-torsion coupling effect

$$
\alpha_{\mathrm{TH}(\mathrm{i})}=1+\left(\frac{\beta}{p_{\mathrm{a}} D}\right) \frac{H_{\mathrm{si}}}{\delta} .
$$

Because lumped formulations are used in the approach, $H_{\mathrm{si}} / \delta$ is the average soil reaction on the pile element whose central point is $i$, denoted as $p_{\mathrm{i}}$. If the two sides of Eq. (17) are divided by $\pi D^{2} \delta / 2, \alpha_{\mathrm{TH}(\mathrm{i})}$ can be expressed in a stress form as,

$$
\alpha_{\mathrm{TH}(\mathrm{i})}=\frac{\bar{\tau}_{\mathrm{s}}}{\bar{\tau}_{\mathrm{s}}^{\prime}}=1+\left(\frac{\beta}{p_{\mathrm{a}} \mathrm{D}}\right) p_{\mathrm{i}}
$$

where $\bar{\tau}_{\mathrm{s}}$ and $\bar{\tau}_{\mathrm{s}}^{\prime}$ are the average torsional shear stresses at the pile element on the group pile and the single pile, respectively. In Eq. (20), $\beta$ quantifies the contribution of subgrade reaction to the increase in the torsional shear resistance at the soil-pile interface. Figure 3 illustrates the physical meaning of $\beta$.

$f_{\mathrm{ij}}^{\mathrm{HH}}$ and $f_{\mathrm{ij}}^{\mathrm{TT}}(i \neq j)$

$f_{\mathrm{ij}}^{\mathrm{HH}}(i \neq j)$, the flexibility coefficient due to the lateral interaction between two piles, is obtained from Mindlin (1936)'s solutions for the influence of a unit lateral point force in a homogeneous, isotropic elastic half-space. This technique has been used by Poulos (1971), Leung and Chow (1987) and others for the analysis of laterally loaded pile groups. Non-homogeneous soils can be tackled by means of an averaging procedure described by Chow (1986). However, for the case of pile groups subjected to torsion, the angle between the lateral loads on two arbitrary piles could be of any value, so a more general analysis of lateral interaction between two piles is needed.

Based on Mindlin's solutions, the lateral displacement of a given point in an arbitrary direction due to a unit lateral load in an interior elastic half space is derived in APPENDIX. In the present approach, the soil flexibility is condensed at the nodes of the pile elements, so the soil displacement at node $i$ on a pile due to a unit lateral force at node $j$ on another pile, $f_{\mathrm{ij}}^{\mathrm{HH}}(i \neq j)$, therefore, can be calculated using Eq. (A5),

$$
f_{\mathrm{ij}}^{\mathrm{HH}}=u_{\mathrm{j}} \cos \gamma_{\mathrm{ij}}+v_{\mathrm{j}} \sin \gamma_{\mathrm{ij}} \quad(i \neq j)
$$

where $u_{\mathrm{j}}$ and $v_{\mathrm{j}}$ are the soil displacements at node $j$ on a pile in the same direction and in the perpendicular direc- 
tion of the unit force at node $i$, respectively; $\gamma_{\mathrm{ij}}$ is the angle between the directions of the two lateral forces at node $i$ and node $j$.

$f_{\mathrm{ij}}^{\mathrm{TT}}(i \neq j)$ represents the torsional component of soil deformation at node $i$ on a pile due to a unit torsional force at node $j$ on another pile. This effect is omitted in this paper since both Poulos (1975) and Kong (2006) found that the interaction between two three-diameter spaced piles, if any, is negligible.

$f_{\mathrm{ij}}^{\mathrm{HT}}$ and $f_{\mathrm{ij}}^{\mathrm{TH}}(i \neq j)$

$f_{\mathrm{ij}}^{\mathrm{HT}}(i \neq j)$ represents the lateral component of soil deformation at node $i$ due to a unit torsional force at node $j$ on another pile. $f_{\mathrm{ij}}^{\mathrm{TH}}(i \neq j)$ represents the torsional component of soil deformation at node $i$ due to a unit lateral force at node $j$ on another pile. $f_{\mathrm{ij}}^{\mathrm{HT}}(i \neq j)$ can be calculated using Randolph (1981)'s analytical solution for torsionally loaded single piles. In Randolph's solution, the soil was treated as independent horizontal layers, so, at a particular depth, the relationship between the circumferential soil displacement at a point, $\rho$, and the torsional shear stress on the soil-pile interface $\tau$ is

$$
\frac{\partial}{\partial r}\left(\frac{\rho}{r}\right)=\frac{\tau D^{2}}{4 G_{s} r^{3}}
$$

where $r$ is the distance from the centre of pile to the point $(r \geq D / 2) ; G_{\mathrm{s}}$ is the shear modulus of soil. Integrating the above equation yields the circumferential soil displacement at the point,

$$
\rho=\frac{\tau D^{2}}{8 G_{\mathrm{s}} r}
$$

In the present approach, torsional shear stresses on pile elements are condensed at nodes. Assume the adjacent piles near the pile subjected to torsion follow exactly the free-field soil displacement. Given a lumped torque at node $i, T_{\mathrm{i}}=\pi \bar{\tau}_{\mathrm{s}} D^{2} \delta / 2$, the induced circumferential soil displacement at node $j$ on another pile $\rho_{\mathrm{j}}$ is,

$$
\rho_{\mathrm{j}}=\frac{T_{\mathrm{i}}}{4 \pi G_{\mathrm{s}} \delta \delta}
$$

where $s$ is the center-to-center spacing between the two piles. Thus, $f_{\mathrm{ij}}^{\mathrm{HT}}$ can be expressed as:

$$
f_{\mathrm{ij}}^{\mathrm{HT}}=\frac{\rho_{\mathrm{j}}}{T_{\mathrm{i}}} \sin \psi=\frac{\sin \psi}{4 \pi G_{\mathrm{s}} \delta} \quad(i \neq j)
$$

where $\psi$ is the angle between the line jointing nodes $i$ and $j$ and the direction of the lateral loading at node $j$. Equation (25) is valid only when nodes $i$ and $j$ are in the same depth. Otherwise, $f_{\mathrm{ij}}^{\mathrm{HT}}(i \neq j)$ is zero.

Consider now the influence of the horizontal loading of a pile on the rotation of a neighboring pile. From the reciprocal theorem, the rotation at node $i$ on pile 1 , due to a unit lateral force at node $j$ on pile 2 , must be equal to the soil displacement at node $j$ on pile 2 in the loading direction due to a unit torsional force at node $i$ on pile 1 , that is

$$
f_{\mathrm{ij}}^{\mathrm{TH}}=f_{\mathrm{ij}}^{\mathrm{HT}}=\frac{\sin \psi}{4 \pi G_{\mathrm{s}} \mathrm{s} \delta} \quad(i \neq j)
$$

\section{GOVERNING EQUATION AND CONSTRAINT CONDITIONS OF PILE-SOIL SYSTEM}

The flexibility matrix $\left[F_{\mathrm{s}}\right]$ in Eq. (5) is inverted to give the following stiffness relationship for the soil

$$
\left\{P_{\mathrm{s}}\right\}=\left[K_{\mathrm{s}}\right]\left\{W_{\mathrm{s}}\right\}
$$

where $\left[K_{\mathrm{s}}\right]\left(=\left[F_{\mathrm{s}}\right]^{-1}\right)$ is the soil stiffness matrix. Equilibrium of the interaction forces acting at the pile-soil interface yields

$$
\left\{P_{\mathrm{s}}\right\}=-\left\{P_{\mathrm{p}}\right\}
$$

Assuming no separation between the soil and the piles, the compatibility of the deformations of the soil and the piles yields

$$
\left\{W_{\mathrm{s}}\right\}=\left\{W_{\mathrm{p}}\right\} .
$$

Using Eqs. (3) and (27)-(29), the load deformation relationship of the pile group system is expressed as

$$
\left(\left[K_{\mathrm{p}}\right]+\left[K_{\mathrm{s}}\right]\right)\left\{W_{\mathrm{p}}\right\}=\{Q\} .
$$

In Eq. (30), if $\left[K_{\mathrm{s}}\right]$ is assumed constant, the pile group system becomes linear elastic. If nonlinear $p-y$ and $\tau-\theta$ curves are used, $\left[K_{\mathrm{s}}\right]$ will vary with soil displacements and the system becomes non-linear. It is noted that the soil stiffness matrix $\left[K_{\mathrm{s}}\right]$ obtained should be augmented with zeros in appropriate rows and columns corresponding to the rotational degrees-of-freedom of the pile to be compatible with the pile stiffness matrix $\left[K_{\mathrm{p}}\right]$.

In this paper, the pile cap in a pile group is assumed rigid and the pile-cap connection is assumed fixed, so rotation of all the pile heads in the vertical plane should be zero, and the twist of all the pile heads should be equal to the twist of the pile cap. The pile-head lateral displacement of an individual pile is equal to the twist angle of the pile group times the distance from the pile to the torsional centre of the group. In addition, the force equilibrium of the pile cap should be satisfied:

$$
\sum_{\mathrm{I}=1}^{\mathrm{N}}\left(T_{\mathrm{I}}+H_{\mathrm{I}} S_{\mathrm{I}}\right)=T_{\mathrm{g}}
$$

where $T_{\mathrm{I}}$ and $H_{\mathrm{I}}$ are the pile-head torque and shear force on pile $I ; S_{\mathrm{I}}$ is the distance between the torsional centre of the pile group to pile $I ; T_{\mathrm{g}}$ is the applied torque on the pile cap; $N$ is the total number of piles in the pile group.

An iteration technique is used to solve Eq. (30). First, assume an initial value of $\left[W_{\mathrm{p}}\right]$, denoted as $\left[W_{\mathrm{p}}\right]^{(0)}$. Then, substitute the initial value into $\left[K_{\mathrm{s}}\right]$ and solve Eq. (30) to obtain the first iterative solution. After that, the diagonal elements of $\left[K_{\mathrm{s}}\right]$ are calculated with the secant moduli of soil subgrade reaction with respect to the first solution of [ $\left.W_{\mathrm{p}}\right]$. Repeating the steps $m$ times, the $m$ th iterative solution is

$$
\left[W_{\mathrm{p}}\right]^{(\mathrm{m})}=\left(\left[K_{\mathrm{p}}\right]+\left[K_{\mathrm{s}}\right]^{(\mathrm{m}-1)}\right)^{-1}\{Q\}
$$


where $\left[K_{\mathrm{s}}\right]^{(\mathrm{m}-1)}=\left[K_{\mathrm{s}}\left(W^{(\mathrm{m}-1)}\right)\right]$. The convergence criterion is

$$
\frac{\sum_{\mathrm{i}=1}^{\mathrm{n}} \boldsymbol{\varpi}_{\mathrm{pi}}^{\mathrm{m}}-\sum_{\mathrm{i}=1}^{\mathrm{n}} \boldsymbol{\varpi}_{\mathrm{pi}}^{\mathrm{m}-1}}{\sum_{\mathrm{i}=1}^{\mathrm{n}} \boldsymbol{\varpi}_{\mathrm{pi}}^{\mathrm{m}-1}}<\varepsilon
$$

where $\boldsymbol{\varpi}_{\text {pi }}$ is the pile deformation at node $i$ and $\varepsilon$ is the allowable tolerance, which is taken as $10^{-3}$ in the numerical calculation. When two consecutive iterative solutions satisfy a convergence criterion, the iteration is terminated. The iteration technique is convergent as long as the $p-y$ curves and $\tau-\theta$ curves are convex. An in-house program, NATLPG, was developed using Mathematica 5 for the present approach.

\section{COMPARISON OF NUMERICAL AND EXPERIMENTAL RESULTS}

\section{Centrifuge Model Tests}

Results of the centrifuge model tests reported by Kong (2006) and Kong and Zhang (2007b) are utilized to verify the proposed approach. Aluminum model piles $19 \mathrm{~mm}$ in diameter and $300 \mathrm{~mm}$ in length were employed in the centrifuge model tests. The model piles were rigidly connected to aluminum pile caps at three-diameter spacing. Three pile group configurations, $1 \times 2,2 \times 2$ and $3 \times 3$, were tested as shown in Fig. 4. Before starting the centrifuge, each of the pile groups was first jacked into the sand bed to $90 \mathrm{~mm}$ using a pile jacking device. After the centrifuge was gradually accelerated to the desired g level, the pile group was jacked at $1 \mathrm{~mm} / \mathrm{s}$ to the final embedment depth of $270 \mathrm{~mm}$. Then, the pile group was torsionally loaded in increments using two horizontal actuators. At each load level, the load was kept constant until no further variations in twist angle were observed. Four laser sensors were used to measure the movements of the pile cap at four points. Any three of the four displacement measurements could be used to calculate the twist angle and the two horizontal displacements of the pile

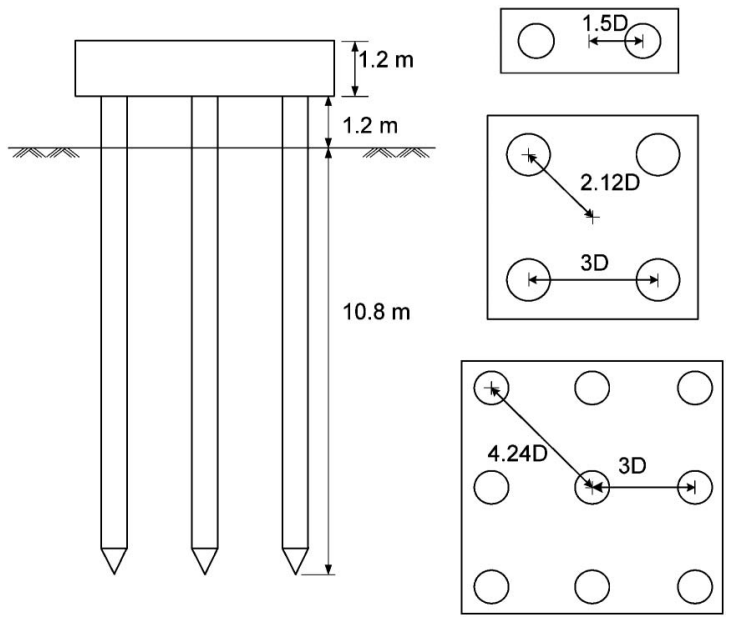

Fig. 4. Pile group dimensions and configurations (not to scale) cap. These tests were conducted at $40 \mathrm{~g}$, thus the model pile groups simulated three-diameter spaced closed-end pipe pile groups with a prototype outside diameter of $0.76 \mathrm{~m}$ and an embedded pile length of $10.8 \mathrm{~m}$, as shown in Fig. 4. The flexural stiffness of piles is $220.5 \mathrm{MN} . \mathrm{m}^{2}$, the Poisson's ratio is 0.3 , and the torsional rigidity of the piles is $169.9 \mathrm{MN} . \mathrm{m}^{2}$. The simulated prototype pile caps are $1.2 \mathrm{~m}$ thick and $1.2 \mathrm{~m}$ above the ground surface in prototype. Details of the pile group tests have been described by Kong and Zhang (2007b).

Leighton Buzzard sand was used in these tests. The sand was a quartz-based uniform sand, with the grain size ranging from 0.09 to $0.15 \mathrm{~mm}$. The average grain size, which is the grain diameter at which $50 \%$ of the soil is finer by weight, was $0.14 \mathrm{~mm}$. Two dry soil densities $\left(13.76 \mathrm{kN} / \mathrm{m}^{3}\right.$ and $\left.14.83 \mathrm{kN} / \mathrm{m}^{3}\right)$ were used, with relative densities of $35 \%$ for the loose sand and $75 \%$ for the dense sand after considering the settlement of the sand bed at the $40 \mathrm{~g}$ acceleration.

\section{Comparison of Numerical and Test Results}

Two sets of soil parameters are required. One set is the soil subgrade reaction parameters for calculating $f_{\mathrm{ii}}^{\mathrm{HH}}$ and $f_{\text {ii }}^{\text {TT }}$; another set is soil modulus, Poisson's ratio and shear modulus of soil for calculating $f_{\mathrm{ij}}^{\mathrm{HH}}, f_{\mathrm{ij}}^{\mathrm{HT}}$ and $f_{\mathrm{ij}}^{\mathrm{TH}}$.

Kong (2006), based on results of laterally loaded and torsionally loaded single pile tests (Kong and Zhang, 2007a; Zhang and Kong, 2006), proposed a series of exponential $p-y$ curves, hyperbolic $\tau$ - $\theta$ curves, and hyperbolic toe torsional resistance curves:

$$
\begin{aligned}
& \frac{p}{D}=k A_{0} \lambda_{\mathrm{z}}\left(\frac{z}{D}\right)^{0.5}\left(\frac{y}{D}\right)^{0.5} \\
& \tau=\frac{A \tau_{\mathrm{f}} \theta}{A \theta+\tau_{\mathrm{f}}} \\
& T_{\mathrm{t}}=\frac{A_{\mathrm{t}} B_{\mathrm{t}} \theta_{\mathrm{t}}}{A_{\mathrm{t}} \theta_{\mathrm{t}}+B_{\mathrm{t}}}
\end{aligned}
$$

where $k$ is a fitting coefficient, $0.01 ; z$ is the embedded depth; $A_{0}$ is a factor related to soil density and soil stress states; $\lambda_{\mathrm{z}}$ is a reduction factor for considering the effect of ground surface; $T_{\mathrm{t}}$ is the toe torsional resistance; $\theta_{\mathrm{t}}$ is the local twist angle of the pile toe; $A$ and $A_{\mathrm{t}}$ are the initial slopes of a hyperbolic $\tau$ - $\theta$ curve and a hyperbolic toe resistance curve, respectively; and $\tau_{\mathrm{f}}$ and $B_{\mathrm{t}}$ are the ultimate shaft torsional shear stress and toe torsional resistance, respectively. Factors $A_{0}, \lambda_{\mathrm{z}}, A, A_{\mathrm{t}}$, and $B_{\mathrm{t}}$ are expressed as,

$$
\begin{aligned}
& A_{0}=2 G_{0}\left(1+v_{0}\right) \frac{\left(2.97-e_{0}\right)^{2}}{1+e_{0}}\left(\frac{2 K_{0}+1}{3} D \gamma_{0}^{\prime} p_{\mathrm{a}}\right)^{0.5} \\
& \lambda_{\mathrm{z}}=\left(\frac{z}{z_{\mathrm{c}}}\right)^{0.5}\left(z<z_{\mathrm{c}}\right) \\
& A=2 c G_{0} \frac{\left(2.97-e_{0}\right)^{2}}{1+e_{0}}\left(\frac{2 K_{0}+1}{3} D \gamma_{0}^{\prime} p_{\mathrm{a}}\right)^{0.5} \\
& A_{\mathrm{t}}=\frac{2}{3} D^{3} G_{\mathrm{t}}
\end{aligned}
$$



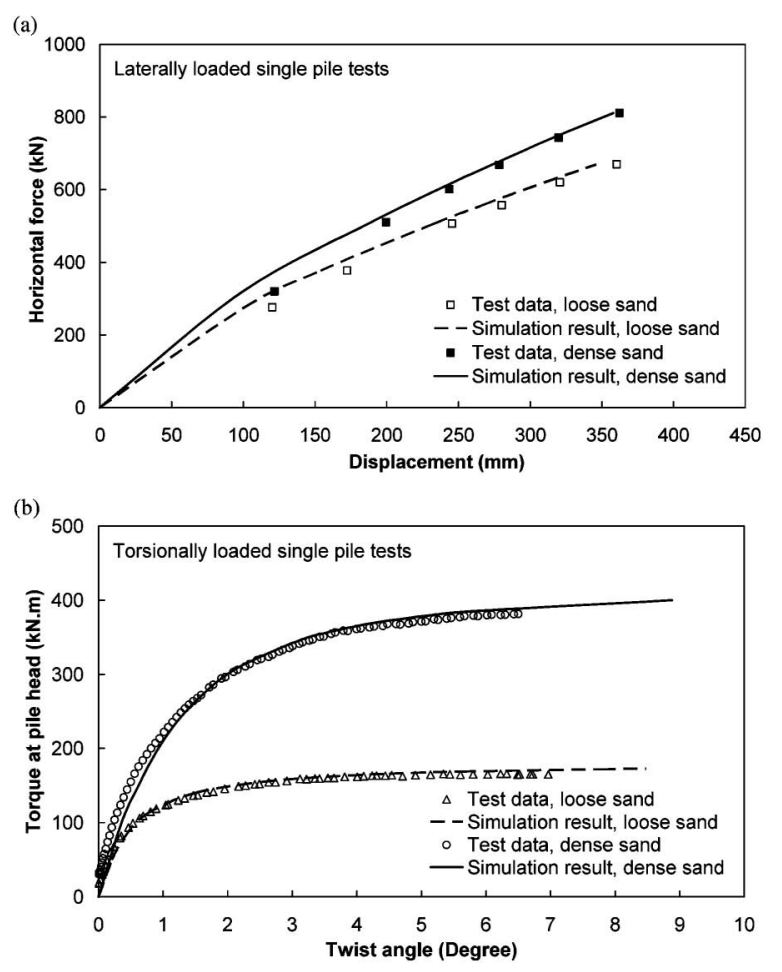

Fig. 5. Comparison of single pile tests and numerical analyses

$$
B_{\mathrm{t}}=\frac{1}{12} \pi D^{3} \tau_{\mathrm{tf}}
$$

where $G_{0}$ is a material parameter, taken as 387 , which is the average value for the Leighton Buzzard sand measured by Cai (2001) with the maximum discrepancy within $10 \% ; v_{0}, e_{0}$ and $\gamma_{0}^{\prime}$ denote the initial Poisson's ratio, initial void ratio, and effective unit weight of sand before pile jacking, respectively; $K_{0}$ is the coefficient of earth pressure at rest, recommended to be 0.4 for loose sand and 0.5 for dense sand by Terzaghi and Peck (1948); $z_{\mathrm{c}}$ is the critical depth, Baguelin et al. (1978) found the critical depth for granular soil is of the order of $4 D ; \lambda_{\mathrm{z}}$ is 1.0 when $z$ is larger than $z_{\mathrm{c}} ; c$ is an empirical factor; and $G_{\mathrm{t}}$ and $\tau_{\mathrm{tf}}$ are the initial shear modulus of pile toe-soil interface and the ultimate torsional shear stress at the pile toe, respectively. $c, \tau_{\mathrm{f}}, G_{\mathrm{t}}$ and $\tau_{\mathrm{tf}}$ are curve-fitted from the experimental $\tau-\theta$ curves and the toe torsional resistance curves reported by Zhang and Kong (2006). Figure 3 shows the schematic shape of the $p-y$ and $\tau-\theta$ curves; further details of the $p-y$ curves, $\tau-\theta$ curves, and the toe torsional resistance curves are described by Kong (2006). Figure 5 compares the simulated and measured horizontal force-displacement curves and torque-twist angle curves for the single piles, which demonstrates the validation of the proposed load transfer curves. Some key parameters for the $p-y$ curves, the $\tau-\theta$ curves, and the toe torsional resistance curves used to simulate the single pile tests, as well as the pile group tests, are summarized in Table 1. Distributions of $c$ and $\tau_{\mathrm{f}}$ are available from Kong (2006).

The shear modulus of soil is a key parameter for pile group interactions. It is calculated from the soil modulus
Table 1. Key parameters for $p-y$ curves, $\tau-\theta$ curves, and toe torsional resistance curves

\begin{tabular}{lccccccr}
\hline & $v_{0}$ & $e_{0}$ & $\begin{array}{c}\gamma_{0}^{\prime} \\
\left(\mathrm{kN} / \mathrm{m}^{3}\right)\end{array}$ & $K_{0}$ & $\begin{array}{c}p_{\mathrm{a}} \\
(\mathrm{kPa})\end{array}$ & $\begin{array}{c}G_{\mathrm{t}} \\
(\mathrm{MPa})\end{array}$ & $\begin{array}{c}\tau_{\mathrm{tf}} \\
(\mathrm{kPa})\end{array}$ \\
\hline Loose sand & 0.2 & 0.897 & 13.76 & 0.4 & 101.3 & 123 & 509 \\
Dense sand & 0.3 & 0.761 & 14.83 & 0.5 & 101.3 & 282 & 2192 \\
\hline
\end{tabular}

and Poisson's ratio. The modulus of cohesionless soils proposed by Poulos (1971) is used in the study. The proposed values of soil modulus are 0.9-2.1 MPa with an average of 1.7 MPa for loose sand, 2.1-4.1 MPa with an average of 3.5 MPa for medium dense sand, and 4.1-9.7 $\mathrm{MPa}$ with an average of $6.9 \mathrm{MPa}$ for dense sand. As pointed out by Poulos (1971), the use of a constant value of soil modulus with depth in sands is highly questionable, and it must be considered as being somewhat artificial fitting parameters rather than meaningful soil modulus. The moduli for the loose and dense sands are taken as 2.1 $\mathrm{MPa}$ and 5.0 $\mathrm{MPa}$ in this study, respectively. For the soil Poisson's ratio, Budhu (2000) suggested typical values from 0.15 to 0.25 for loose sand and from 0.25 to 0.35 for dense sand. In this study, the Poisson's ratios of 0.2 and 0.3 are used for the loose and dense sands, respectively.

Kong and Zhang (2008) reported values of coupling coefficient $\beta$ of 0.4 and 0.8 for the loose and dense sands, respectively, which were back calculated from the $1 \times 2$ pile group tests in the loose and dense sands and the $2 \times 2$ pile group test in the dense sand.

In the present study, each pile is divided into 40 elements. An investigation of the influence of element number found that the use of twenty elements generally produces reasonable accuracy and there is little difference in solutions using forty and fifty elements.

Figure 6 shows the experimental torque-twist angle curves of $1 \times 2,2 \times 2$, and $3 \times 3$ pile groups subjected to torsion and the corresponding numerical predictions using the present approach. Good agreement with the test data is achieved for the $1 \times 2$ and $2 \times 2$ pile groups. For the $3 \times 3$ pile group, agreement is favorable at the initial loading stages, whereas the analysis over-predicts the pile resistance at higher loading stages. In addition, the program was employed to calculate the responses of the pile groups without considering the pile-soil-pile interactions and the deflection-torsion coupling effect. This calculation was performed by setting a large far-field soil modulus, $10^{3} \mathrm{MPa}$, and a $\beta$ value of zero. Kong (2006) found that when the far-field soil modulus is larger than 10 $\mathrm{MPa}$, the effect of the pile-soil-pile interactions on the pile group response is negligible. The calculated results are also shown in Fig. 6. It is found that the curves without considering the pile-soil-pile interactions and the deflection-torsion coupling effect are slightly lower than the present numerical results considering all the interaction and coupling effects for the $1 \times 2$ and $2 \times 2$ pile groups, but are slightly higher for the $3 \times 3$ pile group. Effects of pile-soil-pile interactions and coupling effect 
(a)

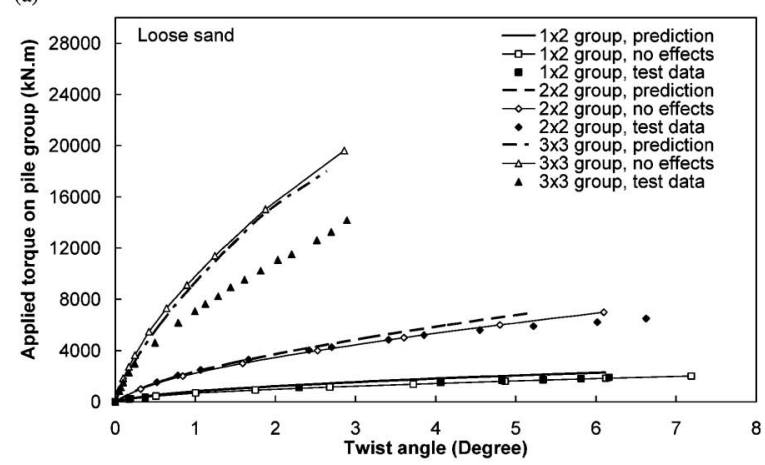

(b)

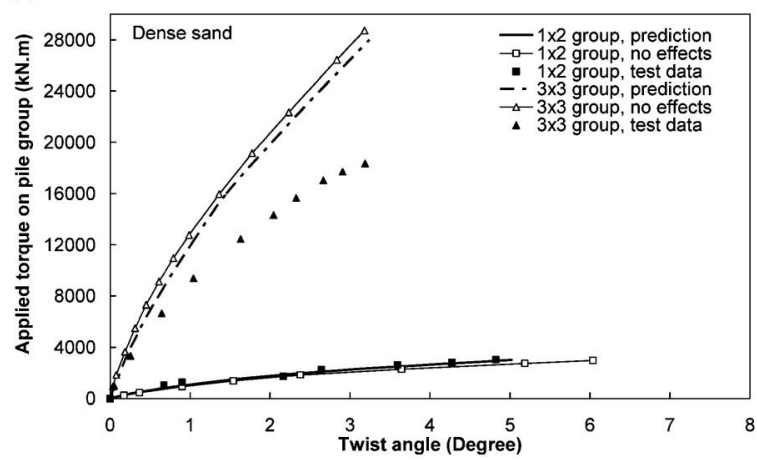

Fig. 6. Torque-twist angle curves of $1 \times 2,2 \times 2$ and $3 \times 3$ pile groups

will be explained in detail later.

The calculated and experimental torsional resistances of individual piles in the $1 \times 2$ and $3 \times 3$ pile groups are compared in Fig. 7. In Fig. 7(a), the numerical predictions fit the test data well, especially at large twist angles; while the analysis not considering the interaction and coupling effects appears to underestimate the torsional resistance at large twist angles. The latter analysis curves reach their ultimate values at a twist angle of $4^{\circ}$, which are similar to the torque-twist angle curves for the single piles in Fig. 5. The comparison between the test data and the calculated results demonstrates that the deflectiontorsion coupling effect indeed exists in the pile groups subjected to torsion and coupling coefficient $\beta$ is sufficient to quantify this coupling effect. Figure 7(b) shows the torsional resistances of the individual piles in the $3 \times 3$ pile group. In Fig. 7(b), the numerical predictions fit the test data well at small twist angles, but somewhat deviate from the test data at large twist angles. The center pile in the $3 \times 3$ pile group is only subjected to a torque at the pile head like a single pile subjected to torque only, but its response from the numerical analysis is weaker than that without considering all the effects because the pile-soilpile interactions are taken into account in the numerical prediction.

Figure 8 shows the calculated and experimental pilehead shear forces in the individual piles in the $1 \times 2$ and 3 $\times 3$ pile groups. In Fig. 8(a), the numerical predictions fit the test data very well. In Fig. 8(b), the numerical predictions tend to over-predict the shear forces, but are better than the results without considering the interaction and
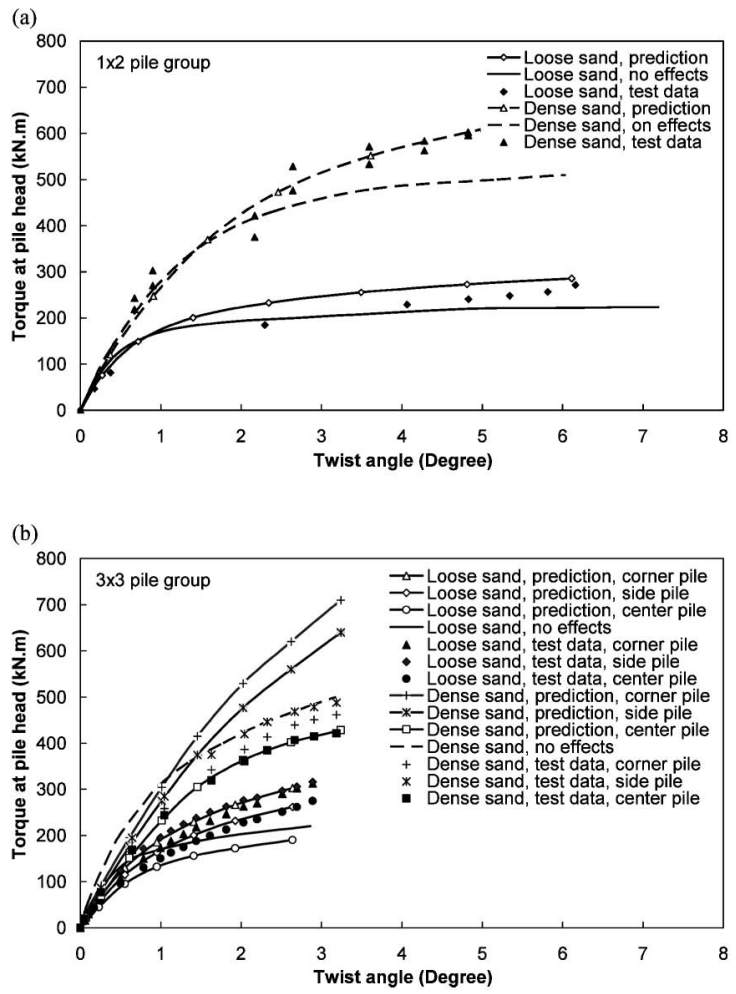

Fig. 7. Torsional resistances of individual piles in $1 \times 2$ and $3 \times 3$ pile groups
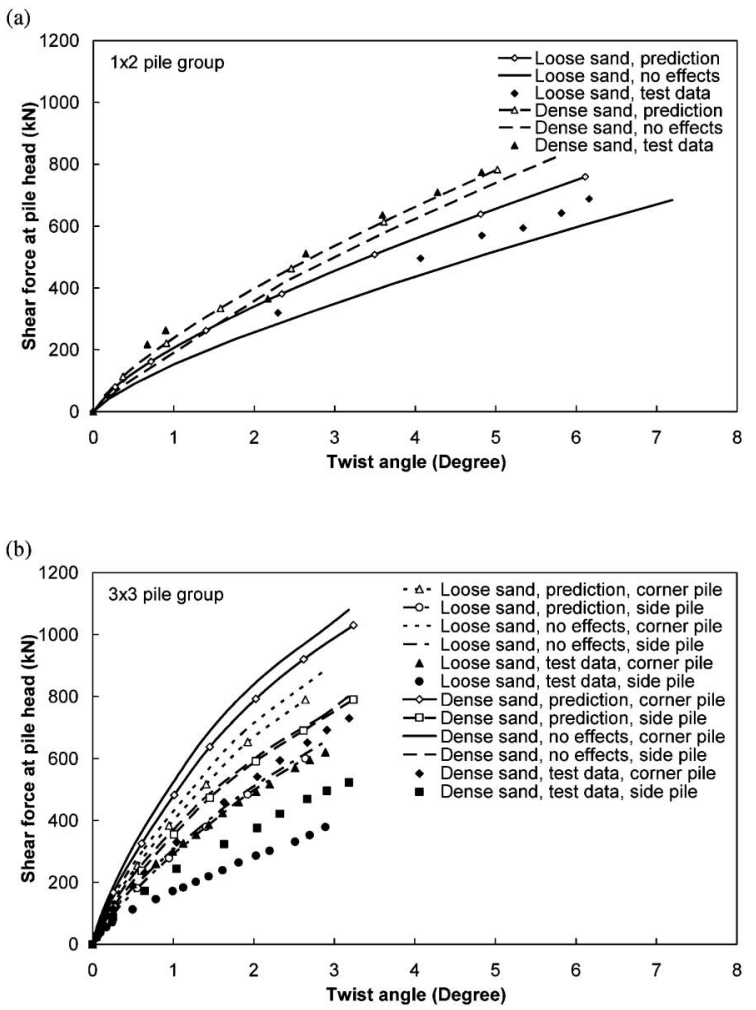

Fig. 8. Pile-head shear forces in individual piles in $1 \times 2$ and $3 \times 3$ pile groups

coupling effects. The difference between Figs. 8(a) and (b) may be attributed to the overlapping of the zones of plas- 
tic flow around the closely spaced individual piles. The plastic flow influences the soil reaction around the pile shafts, and in turn, the torsional resistances shown in Fig. 7(b) through the coupling effect.
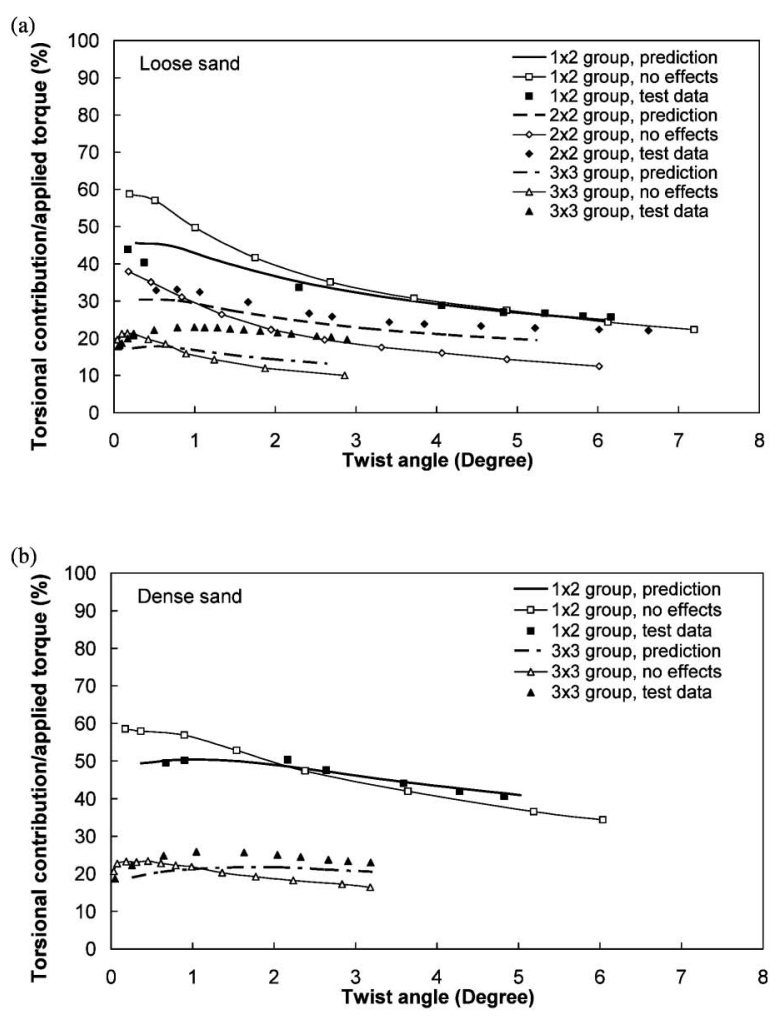

Fig. 9. Percentages of torsional contribution in applied torque
Figure 9 shows the variation of torsional resistance contribution with twist angle, in which the torsional contribution of a pile group is the sum of the torsional resistances of all the individual piles in the pile group. In Fig. 9, the numerical predictions fit the torsional contribution well over the entire range of twist angle for the $1 \times$ 2 and $2 \times 2$ pile groups; while for the $3 \times 3$ pile groups, the present approach slightly underestimates the torsional contribution. The results without considering all effects in the pile groups appear to slightly overestimate the torsional contribution at small twist angles but considerably underestimate the torsional contribution at large twist angles.

The numerical predictions for torque and bending moment distributions along the depth using the present approach in the $1 \times 2$ pile group test in dense sand, as an example, are also compared with the test data, and are shown in Fig. 10. A reasonably good agreement between the predictions and measurements is obtained.

\section{EVALUATION OF INTERACTIONS AND COUPLING EFFECTS}

The present approach uses the empirical factor $\beta$ to quantify the deflection-torsion coupling effect and uses the Mindlin (1936) solutions and the Randolph (1981) solution to calculate pile-soil-pile interactions assuming linear elastic soil medium. The influences of the coupling effect and pile-soil-pile interactions on response of pile groups subjected to torsion are evaluated in this section.

Figure 11 shows the deflection-torsion coupling effect with $\beta=0,0.4$, and 0.8 on the responses of the $1 \times 2,2 \times 2$
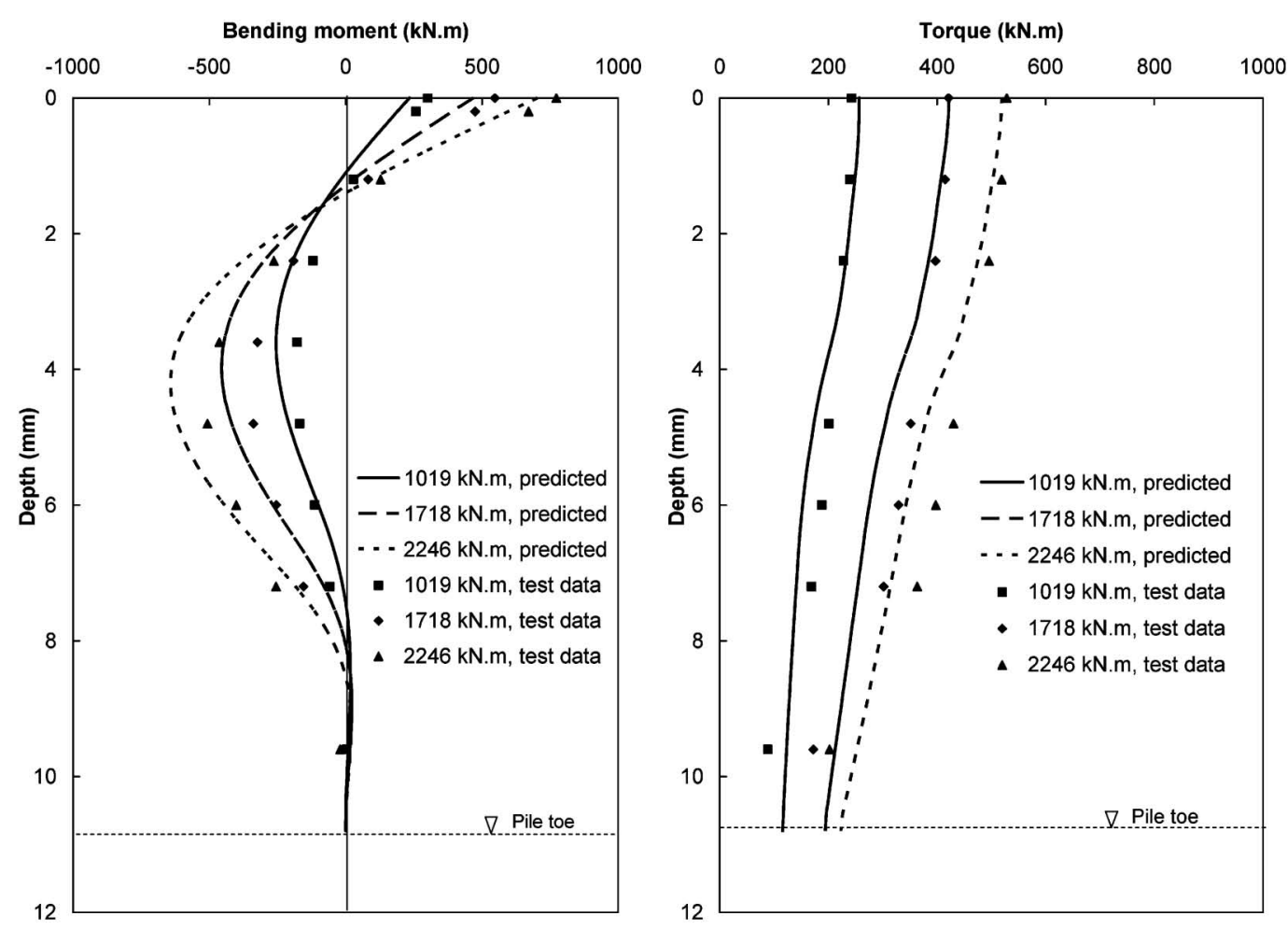

Fig. 10. Distributions of bending moment and torque along depth in $1 \times 2$ pile group in dense sand 


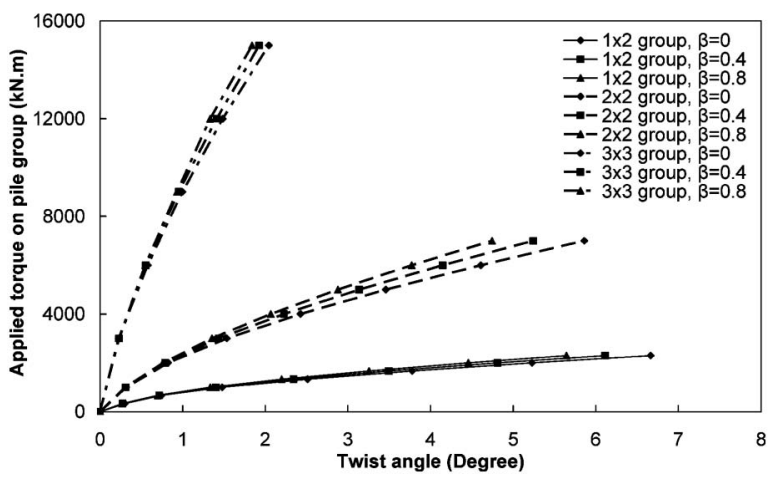

Fig. 11. Deflection-torsion coupling effect on response of pile groups in loose sand (soil modulus 2.1 MPa)

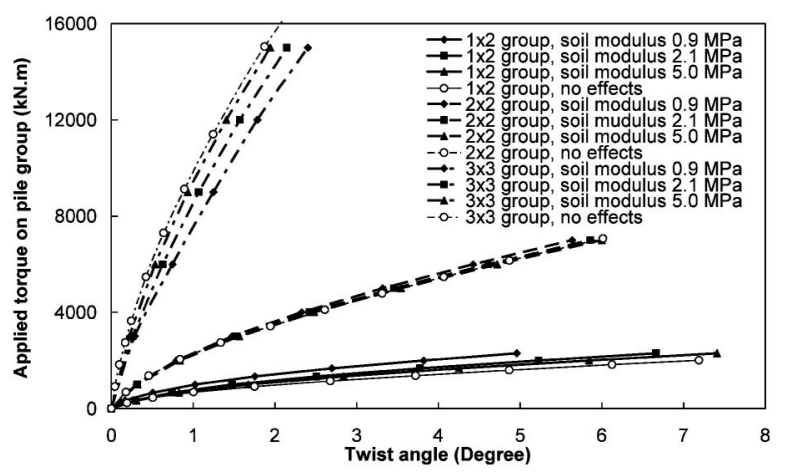

Fig. 12. Pile-soil-pile interaction effects on response of pile groups in loose sand $(\beta=0)$

and $3 \times 3$ pile groups in the loose sand. The group torsional resistance increases with $\beta$ for all the three group configurations. As $\beta$ increases from 0 to 0.8 the group torsional resistance increases by $9-15 \%$. Such an increase is clearly due to the fact that the deflection-torsion coupling effect increases the torsional resistances of individual piles in a pile group.

In the present approach, separate stiffness relationships are used for the near-field soil (i.e., $p-y$ curves and $\tau-\theta$ curves) and for the far-field soil (i.e., pile-soil-pile interactions), which permits independent adjustments of the two relationships. To study the influence of the pile-soilpile interactions on response of pile group subjected to torsion, three far-field soil moduli, 0.9, 2.1 and 5.0 MPa, are used for a parametric study. As reported above, 0.9 $\mathrm{MPa}$ is the lower bound of the recommended soil modulus for loose sand by Poulos (1971). The calculated results are shown in Fig. 12. It is clear that the far-field soil modulus has a significant influence on the response of the pile groups. An increase of soil modulus tends to decrease the torsional stiffness of the $1 \times 2$ and $2 \times 2$ pile groups, but increase the torsional stiffness of the $3 \times 3$ pile group. Because of the linear elastic assumption of soil medium, a movement of a pile in the $1 \times 2$ pile group subjected to torsion induces the second pile to move in the opposite direction. As the far-field soil modulus increases, the backward displacement decreases, so the lateral displace- ments of the two piles in the pile group, and in turn, the torsional stiffness of the pile group decreases. In the $2 \times 2$ pile group, the displacements of a pile induced by the adjacent piles and the pile at the opposite corner counteract, so the influence of far-field soil modulus on the response of the pile group is much smaller than that on the response of the $1 \times 2$ pile group. For a particular pile in the $3 \times 3$ pile group, the interaction with most of the other piles tend to increase its lateral displacement; so the torsional stiffness of the pile group increases as the far-field soil modulus increases. Figure 12 indicates that the pilesoil-pile interactions in pile groups subjected to torsion are sensitive to the group configuration.

In the present approach, the interaction between the lateral resistances of the individual piles and the interaction between the torsional and lateral resistances of the individual piles are quantified separately. A further parametric study indicates that the latter interaction always leads to reduced group torsional stiffness. Therefore, the former and the latter interaction effects counteract in the $1 \times 2$ and $2 \times 2$ pile groups. In the $3 \times 3$ pile group, both types of interaction effects produce a reduced group torsional stiffness.

Experimental results for torsionally loaded pile groups greater than $3 \times 3$ configurations are not yet available. As pile-soil-pile interactions are sensitive to group configuration, more research is needed if the results of this paper are to be extended to larger pile groups subjected to torsion.

\section{SUMMARY}

Previous studies show that piles in a pile group subjected to torsion simultaneously mobilize lateral and torsional resistances. These lateral and torsional resistances induce not only complex pile-soil-pile interactions (i.e., the interactions between the lateral resistances of individual piles and the interaction between the torsional and lateral resistances of the individual piles), but also deflection-torsion coupling effects that the lateral loading has on the torsional resistance of individual piles. In this paper, a nonlinear approach is proposed to predict the response of pile groups subjected to torsion. Nonlinear soil response in the near field is modeled using load-transfer curves (i.e., $p$ - $y$ and $\tau$ - $\theta$ curves). The far-field pile-soilpile interactions are predicted using analytical solutions: the interaction between lateral resistances of the individual piles is considered through Mindlin (1936)'s solutions and the interactions between the torsional and lateral resistances of the individual piles are considered through Randolph (1981)'s solution. An empirical coupling coefficient is proposed to take into account the deflectiontorsion coupling effect. The proposed approach not only simulates nonlinear behavior of individual piles in a pile group but also captures major pile-soil-pile interactions and the coupling effect in the pile group.

The proposed approach was applied to predict results of centrifuge model tests on $1 \times 2,2 \times 2$, and $3 \times 3$ pile groups subjected to torsion. In general, the applied tor- 
que-twist angle response and the transfer of applied torque in the pile groups can be predicted with reasonable accuracy, except for the $3 \times 3$ pile groups where the method tends to underestimate the pile twist angle. In addition, this approach was used to study the influences of pile-soil-pile interactions and the deflection-torsion coupling effect on the response of pile groups. It was found that the deflection-torsion coupling effect can increase the torsional resistance of pile groups by $9-15 \%$. The pile-soil-pile interactions are found to be sensitive to group configuration. More research is needed if the results of this paper are to be extended to pile groups larger than $3 \times 3$ configurations.

\section{ACKNOWLEDGMENTS}

The authors acknowledge the financial support from research grant HKUST 6037/01E provided by the Research Grants Council of Hong Kong SAR and grant 50809060 provided by the National Natural Science Foundation of China.

\section{REFERENCES}

1) Brown, D. A., Morrison, C. and Reese, L. C. (1988): Lateral load behavior of pile group in sand, Journal of Geotechnical Engineering, ASCE, 114(11), 1261-1276.

2) Budhu, M. (2000): Soil Mechanics and Foundations, John Wiley \& Sons, New York.

3) Cai, Z. Y. (2001): A comprehensive study of state-dependent dilatancy and its application in shear band formation analysis, $P h D$ Thesis, The Hong Kong University of Science and Technology, Hong Kong.

4) Chow, Y. K. (1985): Torsional response of piles in nonhomogeneous soil, Journal of Geotechnical Engineering, ASCE, 111(7), 942-947.

5) Chow, Y. K. (1986): Analysis of vertically loaded pile groups, International Journal for Numerical and Analytical Methods in Geomechanics, 10, 59-72.

6) Dutt, R. N. and O'Neill, M. W. (1983): Torsional behavior of model piles in sand, Geotechnical Practices in Offshore Engineering, American Society of Civil Engineers, Austin, 315-334.

7) Georgiadis, M. (1987): Interaction between torsional and axial pile response, International Journal for Numerical and Analytical Methods in Geomechanics, 11, 645-650.

8) Guo, W. D. and Randolph, M. F. (1996): Torsional piles in nonhomogenous media, Computers and Geotechnics, 19(4), 265-287.

9) Hoit, M., Hays, C., McVay, M. and Williams, M. (2001): FB-Pier Users Guide and Manual for the Analysis of Group Pile Foundations, Florida Department of Transportation and the Federal Highway Administration, Tallahassee, Fla, Contract \# DTF61-9500157.

10) Kong, L. G. (2006): Behavior of pile groups subjected to torsion, PhD Thesis, The Hong Kong University of Science and Technology, Hong Kong.

11) Kong, L. G. and Zhang, L. M. (2007a): Rate-controlled lateral-load pile tests using a robotic manipulator in centrifuge, Geotechnical Testing Journal, ASTM, 30(3), 192-201.

12) Kong, L. G. and Zhang, L. M. (2007b): Centrifuge modeling of torsionally loaded pile groups, Journal of Geotechnical and Geoenvironmental Engineering, ASCE, 133(11), 1374-1384.

13) Kong, L. G. and Zhang, L. M. (2008): Experimental study of interaction and coupling effects in pile groups subjected to torsion, Canadian Geotechnical Journal, 45, 1001-1017.

14) Leung, C. F. and Chow, Y. K. (1987): Response of pile groups sub- jected to lateral loads, International Journal for Numerical and Analytical Methods in Geomechanics, 11(3), 307-314.

15) Mindlin, R. D. (1936): Force at a point in the interior of a semi-infinite solid, Physics, 7, 195-202.

16) Poulos, H. G. (1971): Behaviour of laterally loaded piles: II-pile groups, Journal of Soil Mechanics and Foundation Division, ASCE, 97(SM5), 733-751.

17) Poulos, H. G. (1975): Torsional response of piles, The Journal of Geotechnical Engineering Division, ASCE, 101, 1019-1035.

18) Randolph, M. F. (1980): PIGLET: A computer program for the analysis and design of pile groups under general loading conditions, Cambridge University Engineering Department Research Report, Soils TR91.

19) Randolph, M. F. (1981): Piles subjected to torsion, Journal of Soil Mechanics and Foundation Division, ASCE, 107(GT8), 1095-1111.

20) Reese, L. C., Cox, W. R. and Koop, F. D. (1974): Analysis of laterally loaded pile in sand, Proc. 6th Offshore Technology Conference, Houston, TX, paper OTC 2080, 473-483.

21) Reese, L. C., Wang, S. T., Arrellaga, J. A. and Hendrix, J. (2000): Computer Program GROUP for Windows User's Manual, Version 5.0, Ensoft, Austin, Texas.

22) Smith, I. M. (1982): Programming the Finite Element Method, with Application to Geomechanics, Wiley, Chichester.

23) Yan, L. and Byrne, P. M. (1992): Lateral pile response to monotonic head loading, Canadian Geotechnical Journal, 29, 955-970.

24) Zhang, L. M. and Tsang, C. Y. M. (2005): Three-dimensional analysis of torsionally loaded large-diameter bored pile groups, Proc. 6th International Conference on Tall Buildings (eds. by Y. K. Cheung and K.W. Chau), World Scientific, 311-317.

25) Zhang, L. M. and Kong, L. G. (2006): Centrifuge modeling of torsional response of piles in sand, Canadian Geotechnical Journal, 43(5), 500-515.

\section{APPENDIX}

Referring to Fig. A1, given a point $A$ in the half space, the displacements at $A$ in the $X$ - and $Y$-directions, $u$ and $v$, due to a unit point load in the interior of a semi-infinite solid are as follows (Mindlin, 1936):

$$
\begin{aligned}
\left.\begin{array}{rl}
u= & \frac{1}{16 \pi G_{\mathrm{s}}(1-\mu)} \\
& \times\left[\begin{array}{l}
\frac{3-4 \mu}{R_{1}}+\frac{1}{R_{2}}+\frac{x^{2}}{R_{1}^{3}}+\frac{(3-4 \mu) x^{2}}{R_{2}^{3}}+\frac{2 c z}{R_{2}^{3}}\left(1-\frac{3 x^{2}}{R_{2}^{2}}\right) \\
+\frac{4(1-\mu)(1-2 \mu)}{R_{2}+z+c}\left(1-\frac{x^{2}}{R_{2}\left(R_{2}+z+c\right)}\right)
\end{array}\right] \\
v= & \frac{x y}{16 \pi G_{\mathrm{s}}(1-\mu)}\left[\frac{1}{R_{1}^{3}}+\frac{3-4 \mu}{R_{2}^{3}}-\frac{6 c z}{R_{2}^{5}}-\frac{4(1-\mu)(1-2 \mu)}{R_{2}\left(R_{2}+z+c\right)}\right.
\end{array}\right]
\end{aligned}
$$

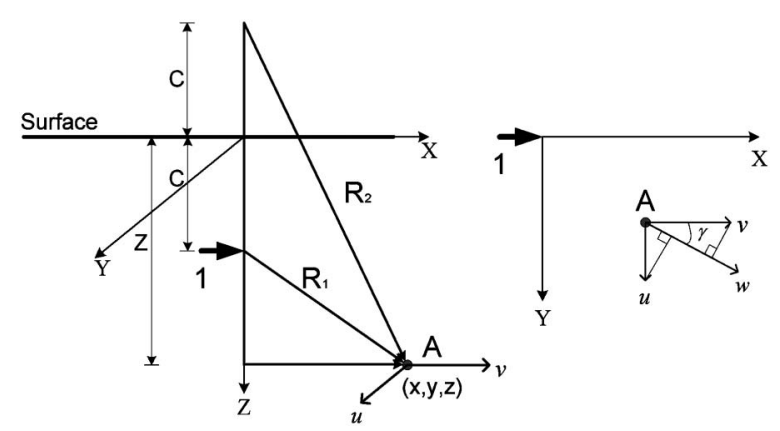

Fig. A1. Unit point load in interior of elastic half-space 
where $z$ is the depth of $A ; G_{\mathrm{s}}$ is the shear modulus of soil; $\mu$ is Poisson's ratio of soil; $c$ is the distance from the ground surface to the loading point; $R_{1}$ and $R_{2}$ are expressed as

$$
R_{1}=\sqrt{r^{2}+(z-c)^{2}}
$$

$$
R_{2}=\sqrt{r^{2}+(z+c)^{2}}
$$

where $r^{2}=x^{2}+y^{2}$. The lateral displacement in any direction, $w$, defined by angle $\gamma$ in Fig. A1, can be calculated by

$$
w=u \cos \gamma+v \sin \gamma
$$

